\title{
An optimization-based approach to extract faceted crystal shapes from stereoscopic images
}

\author{
Stefan Schorsch ${ }^{a}$, Jean-Hubert Hours ${ }^{b}$, Thomas Vetter ${ }^{c, *}$, Marco Mazzotti $^{a}$, \\ Colin N. Jones ${ }^{\mathrm{b}}$ \\ a ETH Zurich, Institute of Process Engineering, Sonneggstrasse 3, CH-8092 Zurich, Switzerland \\ ${ }^{\mathrm{b}}$ EPF Lausanne, Automatic Control Laboratory, EPFL STI IGM LA3 ME C2 398 Station 9, 1015 Lausanne, Switzerland \\ c University of Manchester, School of Chemical Engineering and Analytical Science, Manchester M13 9PL, United Kingdom
}

\section{A R T I C L E I N F O}

\section{Article history:}

Received 1 August 2014

Received in revised form 13 January 2015

Accepted 19 January 2015

Available online 7 February 2015

\section{Keywords:}

Crystallization

Particle shape

Particulate processes

Stereoscopic imaging

Faceted crystals

Nonlinear optimization

\begin{abstract}
A B S T R A C T
The size and shape of particles crucially influences the characteristics of solid products. Until recently these quantities were evaluated using light microscopy. However, extracting the three-dimensional shape of a faceted crystal from a single image is a formidable computer vision challenge. In this work we combine stereoscopic imaging devices (e.g., commercial stereoscopic microscopes or the stereoscopic flow through cell that continuously draws samples from a crystallizer (Schorsch et al., 2014)) with a model-based approach in which parametric polytopes are used to describe faceted crystals (Hours et al., 2014). In the shape reconstruction algorithm these parametric polytopes are scaled and rotated until their projections closely match the measured stereoscopic images, which is formulated as a nonlinear optimization problem. The proposed approach is assessed using simulated images and experimental data. We also assess in which cases the proposed approach does or does not provide advantages over concepts using generic particle shapes (Schorsch et al., 2012).
\end{abstract}

(C) 2015 Elsevier Ltd. All rights reserved.

\section{Introduction}

The particle size and shape distribution (PSSD) that results from a crystallization process influences solid bulk properties that are key factors during downstream processing such as filtration and drying. In the pharmaceutical industry knowledge of the PSSD of an active pharmaceutical ingredient is essential as the API's dissolution behavior and bioavailability critically depend on it (Variankaval et al., 2008; Lovette et al., 2008). The shape of faceted crystals, i.e., their morphology, is the result of the interplay of growth rates of individual crystal facets. Data to estimate crystal size and shape from experiments, either for single crystals or for entire ensembles of particles, can be obtained through imaging systems. Traditionally, crystal growth rates of individual facets are monitored using microscopic observations of stationary single crystals (e.g., Davey et al., 1986; Kitamura and Ishizu, 2000).

Measurement devices that acquire images of a large number of crystals that grow in a stirred tank crystallizer (and therefore in conditions that are closer to an industrial manufacturing process) have recently been developed by different groups and have been

\footnotetext{
* Corresponding author. Tel.: +441613064370.

E-mail address: thomas.vetter@manchester.ac.uk (T. Vetter).
}

used to demonstrate that optical microscopy or video techniques coupled with image analysis can be used to obtain useful crystal shape information. In situ imaging techniques that acquire data directly from the particle suspension in the crystallizer have been presented for example by Roberts and co-workers, who were able to measure two dimensional growth rates (Wang et al., 2007) and to distinguish between different crystal shapes by applying Fourier analysis (Calderon De Anda et al., 2005). Rawlings and coworkers have used in situ imaging combined with model-based object recognition to characterize particles in suspension (Patience and Rawlings, 2001; Larsen et al., 2007). These initial studies motivated a substantial amount of work that has been performed to resolve image segmentation problems, i.e., the problem of overlapping particles (Larsen et al., 2006; Ahmad et al., 2012; Larsen and Rawlings, 2009), which is an important consideration because the number and size of particles during a batch crystallization process usually increases substantially. To circumvent this problem we have recently reported a different approach using an external flow through channel that allowed adjusting the suspension density by diluting with saturated solution drawn from the crystallizer (Eggers et al., 2008a). Moreover, the transparent flow through channel avoided optical distortion effects and depth of field issues that are commonly observed with in situ imaging techniques. Using this setup the evolution of two-dimensional particle size distributions 


$\begin{array}{ll}\text { Notation } & \\ A & \text { matrix of normal vectors } \in \mathbb{R}^{3 \times m}[-] \\ a_{i} & \text { normal vector of the } i^{\text {th }} \text {-facet } \in \mathbb{R}^{3}[-] \\ C & \text { crystal polytope [-] } \\ D_{1 / 2} & \text { dataset } \in \mathbb{R}^{2}[-] \\ d_{i} & \text { 2D data point [-] } \\ M & \text { growth dependency matrix } \in \mathbb{R}^{m \times p}[-] \\ H_{1}, H_{2} & \text { convex hull of a } 2 \text { D dataset [-] } \\ i & \text { index [-] } \\ M & \text { number of vertices of a polytope projection [-] } \\ m & \text { number of facets considered in a model [-] } \\ N & \text { number of points on the convex hull of a projection } \\ & \text { [-] } \\ p & \text { number of independent growth directions [-] } \\ R & \text { rotation matrix } \in S O_{3}(\mathbb{R})[-] \\ s & \text { scaling constant }[\mu \text { m/pixel] } \\ t & \text { scaling vector } \in \mathbb{R}^{p}[-] \\ V_{i} & \text { matrix of vertices of a polytope } \in \mathbb{R}^{3 \times p}[-] \\ D_{i} & \text { dataset } \in \mathbb{R}^{2}[-] \\ x & \text { Cartesian coordinates } \in \mathbb{R}^{2} \text { or } \mathbb{R}^{3}[-] \\ Y & \text { data set } \in \mathbb{R}^{2}[-] \\ d & \text { Hausdorff distance [pixel] } \\ \tilde{d} & \text { averaged Hausdorff distance [pixel] } \\ \epsilon_{\Pi} & \text { reprojection error [-] } \\ P_{1}, P_{2} & \text { 2D projection of a polytope }[-] \\ & \end{array}$

during the cooling crystallization of paracetamol (Kempkes et al., 2010b) and of ascorbic acid (Eggers et al., 2008b) was successfully monitored.

However, all the above-mentioned imaging techniques share a common issue, i.e., they suffer from the orientation dependence that occurs when a complex faceted crystal is projected on a single imaging plane. This issue can be overcome by either using a mathematical model that allows to reconstruct a PSSD from a large set of measured projections by assuming that the particles are randomly oriented (Kempkes et al., 2008; Eggers et al., 2008a) or by obtaining stereoscopic information, i.e., photographing the same particle from two different coplanar directions, therefore enabling the calculation of particle size and shape on a one to one basis. A setup based on external cameras taking pictures of suspended crystals in a reactor has been proposed by Wang et al. (2008), but an experimental implementation has - to the best of our knowledge - yet to be demonstrated in the open literature. Instead, we have presented an improved flow through cell in which particles are photographed from orthogonal directions using two mirrors, thus yielding a quantitative 3D particle size distribution (Kempkes et al., 2010a). In a subsequent work, this cell was connected to a sampling and dilution loop to allow for continuous sampling and real time measurements of PSSDs using improved image analysis routines and particle size calculation protocols (Schorsch et al., 2012). The setup was then further improved in terms of fluid dynamics, image analysis software, and by replacing the mirrors with a second camera yielding higher quality images (Schorsch et al., 2014). Alternatively, if one is only interested in the growth kinetics, where secondary effects (agglomeration and breakage) are not an issue, stereoscopic images of still particles in solution could be obtained, e.g., by using a commercial stereo microscope (with a typical angle of $12-14^{\circ}$ between coplanar observation directions) or a customized microscope installation with a larger angle.

Until recently images obtained via the above-mentioned techniques were interpreted by either using generic particle classes (spheres, cuboids, cylinders, etc.) (Wang et al., 2007; Schorsch et al., 2012) or by assuming that the morphology (i.e., which facets are exhibited on a crystal) of the crystals is known and does not change during an experiment (Calderon De Anda et al., 2005; Larsen et al., 2007), so that only the relative importance of the facets changes. However, the morphology of a crystal evolves during its growth before a "steady state growth morphology" is obtained (Zhang et al., 2006), which requires more flexible approaches to extract size information. Such approaches, in which crystals are described as parametric polytopes, have been presented by Borchert and Sundmacher (2012) and Hours et al. (2014) for monoscopic and stereoscopic setups, respectively.

In this work we follow the approach proposed by Hours et al. (2014) in order to reconstruct faceted crystals from two projections of the same particle. The approach presented in that paper is one that states the computer vision problem as an optimization problem in which the configuration of the polytope (i.e., which facets are present), its scaling and its orientation are optimized such that the difference between projections of the polytope and the measured stereoscopic images is minimized. In Hours et al (2014) we presented some case studies using this approach. In the present study however, we investigate the approach more extensively for a wide variety of particle morphologies and for different crystal systems, using both simulated and experimentally obtained images. We further compare this approach in detail to our previous approach (Schorsch et al., 2014) using generic particles and identify advantages and disadvantages of both approaches in the different cases. In order to investigate if the proposed approach is applicable to commercial hot stage microscopy setups, we investigate the importance of the stereo angle, i.e., the angle between the two coplanar observation directions.

The remainder of this manuscript is structured as follows: First, we briefly describe the particle models used in this work (Section 2) and the data acquisition and image analysis procedures (Section 3); then we explain the reconstruction protocol for the above-mentioned parametric polytopes in Section 4. Finally, the applicability of the method is critically assessed for different stereo angles as well as for different crystal systems and thus different particle morphologies in Section 5.

\section{Particle models}

Faceted crystals can be represented by convex polytopes that can be described from the crystal structure only (unit cell parameters and symmetry) and a list of $m$ experimentally observed facets. Normal vectors $a_{i}$ are calculated for every facet $i$ based on its Miller index. Due to the symmetry of the underlying crystal structure some of the $m$ facets are chemically equivalent, so that they can be grouped into $p$ independent facet groups. This set of normal vectors and constraints can be used to define an inequality which separates the space into two domains. The first is a closed body representing the crystal, which is bounded by planes defined by the normal vectors $a_{i}$ and their normal distance to the origin; while the second domain is the remaining space around the particle. Therefore, according to this definition, a crystal can be expressed as a rotated and scaled polytope $C$ in half space notation:

$C(R, t)=\left\{x \in \mathbb{R}^{3} \mid A R^{T} x \leq M t\right\}$

where $t \in \mathbb{R}^{p}$ is a scaling parameter representing the crystal size for all $p$ independent growth directions as the distance of the bounding plane to the origin, $R$ is a rotation matrix, $A \in \mathbb{R}^{m \times 3}$ is the matrix that contains the normal vectors $a_{i}$ and $M \in \mathbb{R}^{m \times p}$ is a matrix that groups the facets into $p$ independent facet ensembles. Note that the shape of the polytope does not change regardless of the rotation $R$, however, the polytope's projections on the imaging planes are affected and the rotation has therefore to be considered in the optimization problem formulated in Section 4. The basis for defining matrices $A$ 
Table 1

Overview of compounds and crystal forms considered in this paper.

\begin{tabular}{|c|c|c|c|c|c|c|c|}
\hline Substance & Space group & Unit cell & $a[\AA ̊]$ & $b[\AA ̊]$ & $c[\AA]$ & $\beta\left[^{\circ}\right]$ & References \\
\hline Acetaminophen & $\mathrm{P} 2{ }_{1} / a$ & Monoclinic & 12.93 & 9.40 & 7.10 & 115.9 & Hirokawa (1976), Kuvadia and Doherty (2011) \\
\hline Ascorbic acid & $\mathrm{P} 2{ }_{1}$ & Monoclinic & 17.30 & 6.35 & 6.41 & 102.11 & Hvoslef (1968), Srinivasan and Vanitha Devi (2010) \\
\hline Ibuprofen & $\mathrm{P} 2{ }_{1} / c$ & Monoclinic & 14.67 & 7.89 & 10.73 & 99.36 & Shankland et al. (1997), Cano et al. (2001) \\
\hline$\alpha$ L-glutamic acid & $\mathrm{P} 2_{1} 2_{1} 2_{1}$ & Orthorhombic & 7.06 & 10.3 & 8.75 & 90 & Hirokawa (1955), Srinivasan and Dhanasekaran (2011) \\
\hline$\beta$ L-glutamic acid & $\mathrm{P} 2_{1} 2_{1} 2_{1}$ & Orthorhombic & 5.17 & 17.34 & 6.95 & 90 & Hirokawa (1955), Srinivasan and Dhanasekaran (2011) \\
\hline
\end{tabular}

Table 2

Acetaminophen model data.

\begin{tabular}{|c|c|c|c|c|c|c|}
\hline Facet & & $a_{i}$ & & & & \\
\hline$(110)$ & 0.52 & 0.85 & 0 & 1 & 0 & 0 \\
\hline$(1 \overline{1} 0)$ & 0.52 & -0.85 & 0 & 1 & 0 & 0 \\
\hline 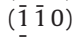 & -0.52 & -0.85 & 0 & 1 & 0 & 0 \\
\hline 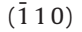 & -0.52 & 0.85 & 0 & 1 & 0 & 0 \\
\hline$(001)$ & 0.26 & 0 & 0.97 & 0 & 1 & 0 \\
\hline$\left(\begin{array}{lll}0 & 0 & 1\end{array}\right)$ & -0.26 & 0 & -0.97 & 0 & 1 & 0 \\
\hline$(20 \overline{1})$ & 0.69 & 0 & -0.72 & 0 & 0 & 1 \\
\hline$(\overline{2} 01)$ & -0.69 & 0 & 0.72 & 0 & 0 & 1 \\
\hline
\end{tabular}

Table 3

Ascorbic acid model data.

\begin{tabular}{|c|c|c|c|c|c|c|}
\hline Facet & & $a_{i}$ & & & & \\
\hline$\left(\begin{array}{lll}1 & 0 & 0\end{array}\right)$ & 1 & 0 & 0 & 1 & 0 & 0 \\
\hline 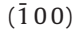 & -1 & 0 & 0 & 1 & 0 & 0 \\
\hline$\left(\begin{array}{lll}0 & 0 & 1\end{array}\right)$ & 0.08 & 0 & 0.99 & 0 & 1 & 0 \\
\hline$\left(\begin{array}{lll}0 & 0 & \overline{1}\end{array}\right)$ & -0.08 & 0 & -0.99 & 0 & 1 & 0 \\
\hline$(10 \overline{1})$ & 0.29 & 0 & -0.96 & 0 & 1 & 0 \\
\hline 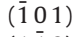 & -0.29 & 0 & 0.96 & 0 & 1 & 0 \\
\hline$\left(\begin{array}{lll}1 & \overline{1} & 0\end{array}\right)$ & 0.35 & -0.94 & 0 & 0 & 0 & 1 \\
\hline$\left(\begin{array}{lll}\overline{1} & 1 & 0\end{array}\right)$ & -0.35 & 0.94 & 0 & 0 & 0 & 1 \\
\hline$\left(\begin{array}{lll}1 & 1 & 0\end{array}\right)$ & 0.35 & 0.94 & 0 & 0 & 0 & 1 \\
\hline$\left(\begin{array}{lll}\overline{1} & \overline{1} & 0\end{array}\right)$ & -0.35 & -0.94 & 0 & 0 & 0 & 1 \\
\hline
\end{tabular}

and $M$ is given by the symmetry of a given crystal system. Even after defining the crystal structure (cf. Section 4) there are - in principle - an infinite number of possible facets that could be exposed for a given crystal form. However, the list of crystal facets that should be considered can be narrowed down by only considering the most stable ones (typically the ones that provide opportunities for strong interactions, e.g., hydrogen bonds, with the solvent molecules) as they are most likely to be present under moderate experimental conditions (see, e.g., Lovette et al. (2008) and Kuvadia and Doherty (2011) for an in depth discussion). In Table 1 we report the structures of the crystal systems considered in this paper where the first reference given in each row reports the crystal structure and the second reference gives a list of stable facets. The matrices $A$ and $M$ can then be reported as in Tables 2-7 for the crystal systems investigated in this work. An example of a crystal polytope can be found in Fig. 1 where a model of an acetaminophen crystal is shown for which the facet families $\{201\},\{110\}$ and $\left\{\begin{array}{lll}0 & 0 & 1\end{array}\right\}$ are shown in blue, red and yellow, respectively. One can easily generate two projections, $P_{1}(R, t)$ and $P_{2}(R, t)$, of the polytope $C$ as it is seen from the previously defined coplanar observation directions. The

Table 4

Cube model data.

\begin{tabular}{lrrrrrr}
\hline Facet & \multicolumn{1}{c}{$a_{i}$} & & \multicolumn{3}{c}{$M$} \\
\hline$\left(\begin{array}{lll}1 & 0 & 0\end{array}\right)$ & 1 & 0 & 0 & 1 & 0 & 0 \\
$\left(\begin{array}{lll}1 & 0 & 0\end{array}\right)$ & -1 & 0 & 0 & 1 & 0 & 0 \\
$\left(\begin{array}{lll}0 & 1 & 0\end{array}\right)$ & 0 & 1 & 0 & 0 & 1 & 0 \\
$\left(\begin{array}{lll}0 & 1 & 0\end{array}\right)$ & 0 & -1 & 0 & 0 & 1 & 0 \\
$\left(\begin{array}{llll}0 & 0 & 1\end{array}\right)$ & 0 & 0 & -1 & 0 & 0 & 1 \\
$\left(\begin{array}{llll}0 & 0 & \overline{1}\end{array}\right)$ & 0 & 0 & -1 & 0 & 0 & 1 \\
\hline
\end{tabular}

Table 5

Ibuprofen model data.

\begin{tabular}{|c|c|c|c|c|c|c|}
\hline Facet & & $a_{i}$ & & & & \\
\hline$\left(\begin{array}{lll}1 & 0 & 0\end{array}\right)$ & 1 & 0 & 0 & 1 & 0 & 0 \\
\hline 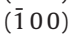 & -1 & 0 & 0 & 1 & 0 & 0 \\
\hline$(001)$ & 0.12 & 0 & 0.99 & 0 & 1 & 0 \\
\hline$(00 \overline{1})$ & -0.12 & 0 & -0.99 & 0 & 1 & 0 \\
\hline (011) & 0.09 & 0.8 & 0.71 & 0 & 0 & 1 \\
\hline$(0 \overline{1} 1)$ & -0.09 & 0.8 & -0.71 & 0 & 0 & 1 \\
\hline$(01 \overline{1})$ & 0.09 & -0.8 & 0.71 & 0 & 0 & 1 \\
\hline$(0 \overline{1} \overline{1})$ & -0.09 & -0.8 & -0.71 & 0 & 0 & 1 \\
\hline
\end{tabular}

Table 6

L-glutamic acid $\alpha$ data.

\begin{tabular}{|c|c|c|c|c|c|}
\hline \multirow{2}{*}{$\frac{\text { Facet }}{\left(\begin{array}{lll}0 & 0 & 1\end{array}\right)}$} & \multicolumn{3}{|c|}{$a_{i}$} & \multicolumn{2}{|c|}{$M$} \\
\hline & 0 & 0 & 1 & 1 & 0 \\
\hline$\left(\begin{array}{lll}0 & 0 & \overline{1}\end{array}\right)$ & 0 & 0 & -1 & 1 & 0 \\
\hline$\left(\begin{array}{lll}1 & 1 & 0\end{array}\right)$ & 0.78 & 0.63 & 0 & 0 & 1 \\
\hline$(1 \overline{1} 0)$ & 0.78 & -0.63 & 0 & 0 & 1 \\
\hline$\left(\begin{array}{lll}1 & 1 & 0\end{array}\right)$ & -0.78 & 0.63 & 0 & 0 & 1 \\
\hline$\left(\begin{array}{lll}\overline{1} & 1 & 0\end{array}\right)$ & -0.78 & -0.63 & 0 & 0 & 1 \\
\hline
\end{tabular}

two projections for the acetaminophen crystal are also reported in Fig. 1.

Depending on the scaling vector $t$ not all of the $m$ facets might be visible on the crystal, so that domains of $t$ with a common set of constituting facets and edges can be identified, which can be accomplished using the analytical expressions reported by Borchert and Sundmacher (2013) and Singh and Ramkrishna (2013) or numerically as shown in Hours et al. (2014). An example of such a morphology map is given in Fig. 2 for the case of acetaminophen. Differently shaded regions represent domains with common facets being present, while black lines separate regions with common edges within such a facet domain. One can thus identify five different crystal morphologies, for which exemplary polytopes are shown in Fig. 3.

In order to compare the results obtained with the polytope representation to the particle sizing technique as demonstrated in previous works, three shape classes, that had been introduced in Schorsch et al. (2012, 2014), will be used as well, i.e., a sphere with its diameter as its only characteristic length, a cylinder with

Table 7

L-glutamic acid $\beta$ data.

\begin{tabular}{|c|c|c|c|c|c|c|}
\hline Facet & & $a_{i}$ & & \multicolumn{3}{|c|}{$M$} \\
\hline$\left(0 \begin{array}{lll}0 & 0\end{array}\right)$ & 0 & 1 & 0 & 1 & 0 & 0 \\
\hline$(0 \overline{1} 0)$ & 0 & -1 & 0 & 1 & 0 & 0 \\
\hline$(021)$ & 0 & 0.89 & 0.45 & 0 & 1 & 0 \\
\hline$(0 \overline{2} 1)$ & 0 & -0.89 & 0.45 & 0 & 1 & 0 \\
\hline$(02 \overline{1})$ & 0 & 0.89 & -0.45 & 0 & 1 & 0 \\
\hline$(0 \overline{2} \overline{1})$ & 0 & -0.89 & -0.45 & 0 & 1 & 0 \\
\hline$\left(\begin{array}{lll}1 & 0 & 1\end{array}\right)$ & 0.80 & 0 & 0.60 & 0 & 0 & 1 \\
\hline$(\overline{1} 01)$ & 0.80 & 0 & -0.60 & 0 & 0 & 1 \\
\hline 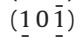 & 0.80 & 0 & -0.60 & 0 & 0 & 1 \\
\hline 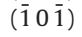 & -0.80 & 0 & -0.60 & 0 & 0 & 1 \\
\hline
\end{tabular}




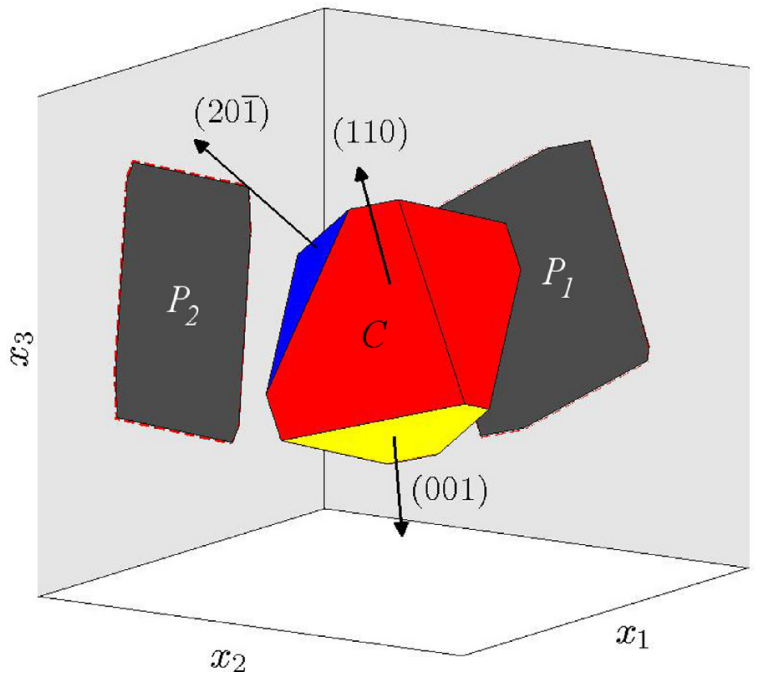

Fig. 1. Projections, fitted contours, and $3 D$ representation of a polytope of a simulated acetaminophen crystal. Projections: dark grey areas, boundary contours: dashed red lines, reconstructed polytope: red, blue, and yellow body. (For interpretation of references to colours in this figure legend, the reader is referred to the web version of this article.)

diameter and length as its two characteristic lengths, and a cuboid with length, width, and depth as its three characteristic lengths.

\section{Image acquisition}

In order to assess the particle sizing techniques, two different data sources are utilized. First, artificially generated images containing projections of faceted crystals are analyzed. These images have the advantage that all aspects of the polytopes in these images, i.e., their orientation $R$ and shape vector $t$, are known and can be used to compare the analysis result to the input. One may also freely choose the angle between the coplanar observation directions. Second, images of crystals from five different organic substances are photographed and investigated. In this case, a different error metric

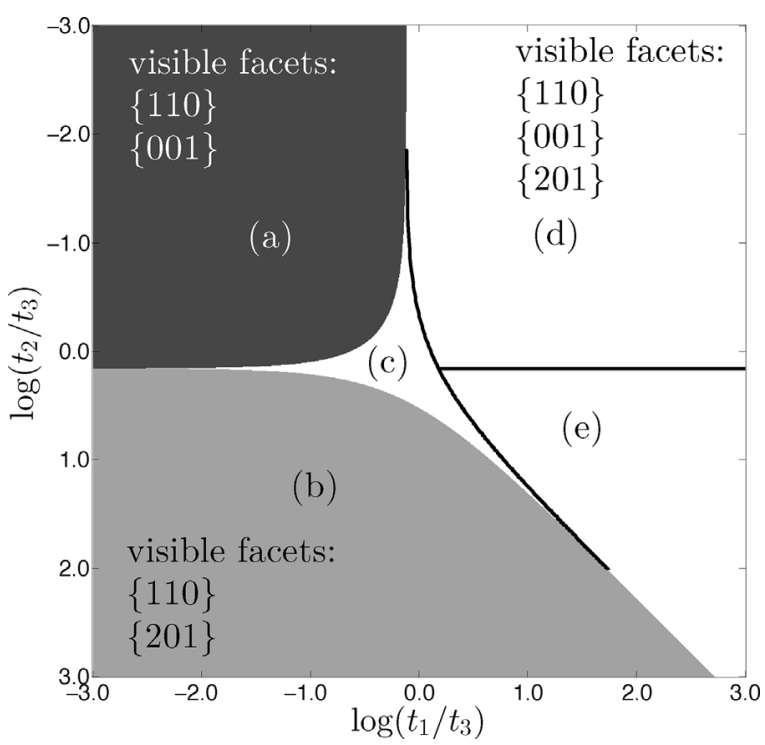

Fig. 2. Morphology map of acetaminophen with facet groups reported in Table 2. Different grey values indicate morphology domains with a common set of constituting facets. Within the white area, two black lines separate regions of different sets of characteristic edges. Example polytopes, indicated as (a)-(e), are shown in Fig. 3.

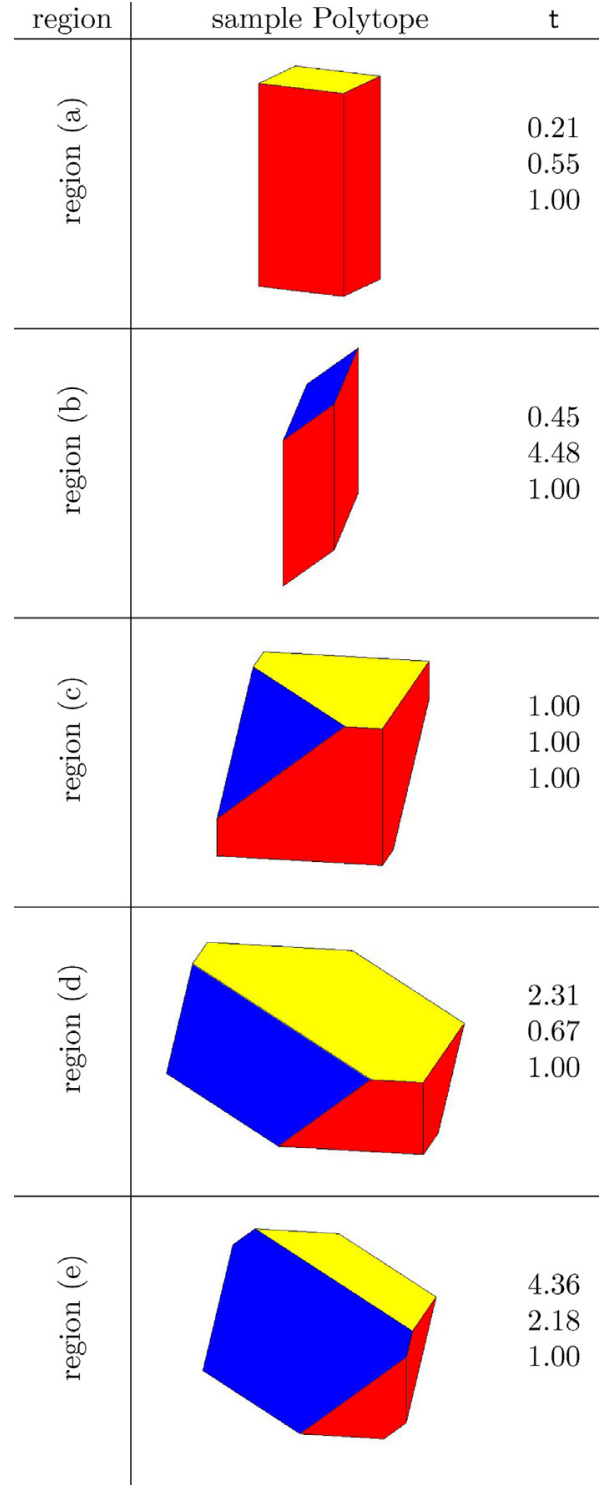

Fig. 3. Sample polytopes of all five morphology regions of acetaminophen corresponding to the regions in Fig. 2 and the scaling vectors used to construct these. The $\{110\}$ family is drawn in red, $\{001\}$ in yellow and $\{20 \overline{1}\}$ in blue. (For interpretation of references to colours in this figure legend, the reader is referred to the web version of this article.)

(see Section 5.1) is necessary as neither the rotation nor the size vector are known a priori.

\subsection{Generation of artificial images}

Simulated projections of crystals are generated from the polytope model of the crystal using the Multi-Parametric Toolbox (MPT 3.0 (Kvasnica et al., 2012)) available for Matlab (MATLAB, 2010). The projections are then converted to images with the same resolution as our measurement setup using the poly2mask function available in the Image Processing Toolbox of Matlab.

\subsection{Stereoscopic imaging device}

Crystals in suspension are photographed from two orthogonal coplanar directions using our custom built measurement device which is described and characterized in detail in Schorsch et al. (2014). In short, two cameras take pictures of crystals in 

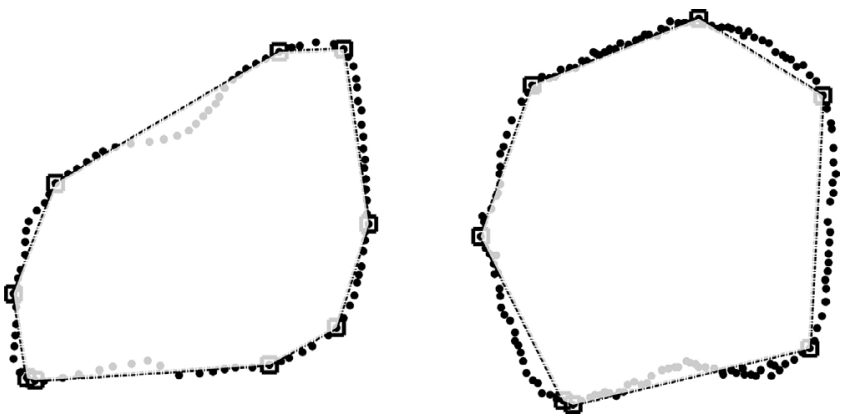

Fig. 4. Application of the Douglas-Peucker algorithm to a pair of images of an acetaminophen crystal. The points extracted from the images appear as black dots. The black squares correspond to the vertices of the polygonal line produced by the Douglas-Peucker algorithm. The convex polygon corresponds to the convex hull of the output points of the Douglas-Peucker procedure.

suspension in a flow through cell made of sapphire glass. The crystals are conveyed to this cell and fed back into a crystallizer using a sampling loop. The projections drawn into the $\left(x_{1}, x_{3}\right)$ and $\left(x_{2}\right.$, $x_{3}$ ) planes in Fig. 1 represent the information captured by the two cameras as a (simulated) example of a crystal as it passes through the measurement cell.

\subsection{Image analysis}

The image analysis algorithm is the same for the artificially generated images of crystals as well as the actual photographs. It is based on OpenCV (Bradski, 2000). The details of this procedure are described in (Schorsch et al., 2012, 2014). After thresholding, contour extraction, and comparing both projections with respect to their $x_{3}$ position a matching pair of projections is obtained, whose sets of boundary points $D_{1}$ and $D_{2}$ are then extracted:

$D_{1}=\left\{d_{1, i}\right\}_{i=1}^{N_{1}}$ and $D_{2}=\left\{d_{2, i}\right\}_{i=1}^{N_{2}}$

where $D_{1}$ is a dataset of coordinates of $N_{1}$ boundary points of $P_{1}$, and $D_{2}$ is a data set of coordinates of $N_{2}$ boundary points of $P_{2}$, where we have used the shorter notation $P_{i}$ for $P_{i}(R, t)$, as always in the following. For further analysis, the convex hulls, $\mathrm{H}_{1}$ and $\mathrm{H}_{2}$, of these two data sets are calculated via a Graham scan (Graham, 1972). In order to reduce the number of data points and to remove outliers we preprocess the data by employing a Douglas-Peucker line simplification (Douglas and Peucker, 1973). The idea is to identify good candidates for the vertices of the projection. An example for an experimental image of an acetaminophen crystal is presented in Fig. 4. As one can see, this simplification introduces some discrepancy between the data points considered in the optimization algorithm and the actual projection. However, for most projections the simplification leads to good reconstruction results. The optimization algorithm, which will be introduced in the next section, operates on centered and normalized convex hulls, so that $d_{j, i} \in(-1$, 1 ). In order to retrieve the actual size of the particle after the reconstruction is performed, we introduce the normalization constant $s$, which includes the camera magnification and the normalization information.

\section{Optimization-based polytope reconstruction}

The crystal shape estimation problem can be formulated as a polytope reconstruction problem from a pair of projections (Hours et al., 2014). More precisely, the goal is to provide an estimate of the size vector $t$ from two sets of boundary points on projection planes, i.e., from $D_{1}$ and $D_{2}$ defined in Eq. (2). As the projections of a polytope depend strongly on its orientation a good estimate of its size vector $t$ relies on a good estimate of its orientation $R$, so that $t$ and $R$ need to be estimated simultaneously. Moreover, a challenging aspect of the problem is that the solutions are not unique, i.e., more than one pair of $t$ and $R$ can produce the same datasets $D_{1}$ and $D_{2}$.

For estimating both the crystal shape and its orientation, we propose an approach based on minimizing the re-projection error between the rotated and scaled polytope $C$ and the two sets of data points $D_{1}$ and $D_{2}$.

\subsection{Definition of the optimization objective}

The first key ingredient of our method is an appropriate metric to measure the discrepancy between data and scaled model. The metric should be appropriate for optimization purposes and should measure the shape resemblance in a non-ambiguous way, which means that a small value of the optimization objective should correspond to a good fit between the projected and rotated model and the data points. To our knowledge, the Hausdorff distance, $d$, is an appropriate way of measuring the resemblance between a model shape and a shape on a 2D image (Huttenlocher et al., 1993). It is a measure of the distance between two sets, which can be defined as follows:

$d(X, Y)=\max \left\{\operatorname{supinf}_{x \in X}\|x-y\|_{2}, \operatorname{supinf}_{y \in Y}\|x-y\|_{2}\right\}$

where $\|\cdot\|_{2}$ is the Euclidean norm in $\mathbb{R}^{2}, X$ and $Y$ are two (possibly infinite) sets in $\mathbb{R}^{2}$ and sup and inf are the supremum and infimum, respectively. In our case, $X$ is one of the projections of our polytope, $P_{k}$, and $Y$ is the convex hull $H_{k}$ of the measured contour $D_{k}$ for $k \in\{1 ; 2\}$. Hours et al. (2014) have shown that the Hausdorff distance in such cases can be written in a simplified form involving only the vertices $V_{k}$ of the projection $P_{k}$, so that the Hausdorff distance becomes (for $k \in\{1 ; 2\}$ ):

$d_{k}\left(V_{k}, H_{k}\right)=\max \left\{\operatorname{maxmin}_{x \in V_{k}}\|x-y\|_{2}, \operatorname{maxmin}_{y \in H_{k}}\|x-y\|_{2}\right\}$

Note that in Eq. (4) min and max have replaced inf and sup in Eq. (3) since the two sets compared in this equation are non-empty and finite. However, Eq. (4) is not a smooth function and therefore leads to optimization problems that are cumbersome to solve (Lakhtin and Ushakov, 2005). Therefore, we propose to take an averaged version of the Hausdorff distance, which, accounting for both projections now, can be written as:

$\tilde{d}=\sum_{k=1}^{2}\left\{\frac{1}{M_{k}} \sum_{i=1}^{M_{k}} \min _{y \in H_{k}}\left\|x_{i}-y\right\|_{2}+\frac{1}{N_{k}} \sum_{j=1}^{N_{k}} \min _{x \in V_{k}}\left\|x-y_{j}\right\|_{2}\right\}$

where $M_{k}$ is the number of vertices of $P_{k}$ and $N_{k}$ is the number of points in $H_{k}$.

\subsection{Definition and solution of the optimization problem}

The estimates of the size vector $t$ and of the rotation $R$ are taken to be the solutions of the following nonlinear and nonconvex optimization problem:

$\min \tilde{d}$

$t, R$

The rotation matrix is parameterized using quaternions, $q$, which gives (Schmidt and Niemann, 2001; Hours et al., 2014):

$R(q)=\frac{1}{\|q\|_{2}^{2}}\left(r_{1}(q) \quad r_{2}(q) \quad r_{3}(q)\right)$ 


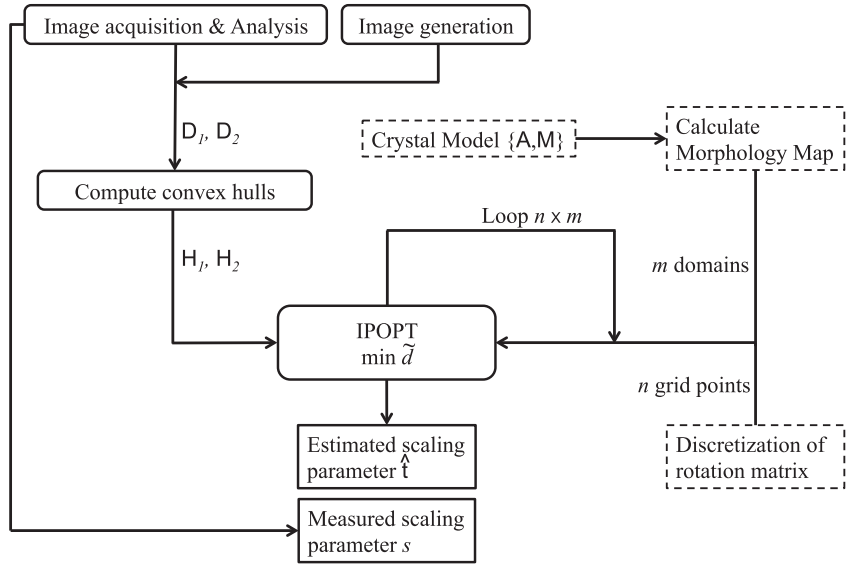

Fig. 5. Schematic overview of the optimization algorithm.

where

$$
\begin{gathered}
r_{1}(q)=\left(\begin{array}{c}
q_{0}^{2}+q_{1}^{2}-q_{2}^{2}-q_{3}^{2} \\
2\left(q_{1} q_{2}+q_{0} q_{3}\right) \\
2\left(q_{1} q_{3}-q_{0} q_{2}\right)
\end{array}\right), \quad r_{2}(q)=\left(\begin{array}{c}
2\left(q_{1} q_{2}-q_{0} q_{3}\right) \\
q_{0}^{2}-q_{1}^{2}+q_{2}^{2}-q_{3}^{2} \\
2\left(q_{2} q_{3}+q_{0} q_{1}\right)
\end{array}\right), \\
r_{3}(q)=\left(\begin{array}{c}
2\left(q_{1} q_{3}-q_{0} q_{2}\right) \\
2\left(q_{2} q_{3}-q_{0} q_{1}\right) \\
q_{0}^{2}-q_{1}^{1}-q_{2}^{2}+q_{3}^{2}
\end{array}\right)
\end{gathered}
$$

It can be shown that $q \neq 0$ and that each element lies within $[-1,1]$ (Hours et al., 2014). Due to the nonlinearity and nonconvexity of the problem, we choose to solve the optimization problem Eq. (6) for every region in the morphology map separately by putting constraints on the scaling vector $t$. These constraints can be precomputed either numerically (as shown in Hours et al., 2014) or analytically (as shown in Borchert and Sundmacher, 2013; Singh and Ramkrishna, 2013). Furthermore, we warm-start a nonlinear solver (IPOPT, see Wächter and Biegler (2006)) from different initial conditions for the rotation matrix $R$. The number of initial points that need to be considered for the rotation matrix in order to arrive at a good reconstruction of the particle morphology is dependent on the complexity of the crystal, its rotation, as well as on the optical properties of the imaging system (the quality of the images). For the cases presented in Section 5 we found that using a linearly spaced grid of $7 \times 7 \times 7$ points (in axis-angle representation (Hornegger and Tomasi, 1999)) is sufficient to get a good reconstruction. We arrived at this conclusion by performing a sensitivity analysis on an exemplary crystal of $\alpha$ L-glutamic acid. Every grid point is given as initial condition to IPOPT, which is run until a specified level of accuracy on a local optimum is met. In the end, the optimal fit is taken to be the best parameter estimate $(\hat{t}, \hat{R})$ resulting in the lowest value of $\tilde{d}$. Fig. 5 gives a schematic overview of the procedure. Thus, for $n$ grid points in the discretization of the rotation matrix and $m$ morphology regions, $n \times m$ optimization problems need to be solved for every pair of measured projections, i.e., for every particle. To get a reconstruction of one typical crystal takes approximately one minute using the presented methodology. The size of the crystal is finally obtained by multiplying $\hat{t}$ with the scaling constant $s$.

\section{Results and discussion}

In order to evaluate the particle sizing procedure a selection of five different organic substances (see Table 1) has been investigated both by generating artificial images and by taking actual photographs of crystals produced in experiments. The substances are acetaminophen, ascorbic acid, Ibuprofen and the $\alpha$ and $\beta$ polymorphs of L-glutamic acid. The selection covers a wide range of different crystal shapes from needle like crystals with a very high aspect ratio to platelets and rather compact crystals with a low aspect ratio. For reconstructing simulated particles, a cuboidal polytope was considered as well.

\subsection{Error metric}

Two different error metrics have been used to evaluate the performance and accuracy of the optimization algorithm. For generated images, the input data in terms of the true size of all facets as the scaling vector $t$ was available, hence the ratio $\hat{t}_{i} / t_{i}$ for each facet $i$ can be calculated. In addition to the recalculation error one can define the reprojection error $\epsilon_{\Pi}$ :

$\epsilon_{\Pi}=\frac{\left\|H_{1} \backslash P_{1}\right\|_{2}+\left\|H_{2} \backslash P_{2}\right\|_{2}}{\left\|P_{1}\right\|_{2}+\left\|P_{2}\right\|_{2}}$

This error measures the difference between the input projection and the calculated reprojection. It can thus be considered a measure of the quality of fit.

\subsection{Analysis of generated images}

As a first step, artificially generated images of acetaminophen crystals belonging to each of the five morphology regions (cf. Fig. 2) have been studied. Secondly, artificial images of the other four crystal systems and of the cuboid model have been analyzed as well. The model parameters in terms of matrices $A$ and $M$ are reported in Tables 2-7. The resulting images have been processed according to Section 3.3 and passed over to the optimization algorithm.

\subsubsection{Fitting of different morphologies of the same crystal}

The first series of simulations is aimed at proving that the optimization algorithm is capable of finding satisfactory results independent of the morphology region where the particle to be analyzed is located. In a case study for acetaminophen, for which the morphology map is reported in Fig. 2, 100 randomly oriented polytopes for each of the five regions have been generated and handed over to the image analysis and size calculation procedures. In Fig. 6, example polytopes for each region (black solid lines in the first column), their projections (dark grey areas), and input scaling vectors $t$ are reported. For each of the randomly selected original polytopes the reconstructed polytope is reported as an overlay in red. As one can see, there is an excellent agreement between input and optimization result. The corresponding fitting contour is plotted on top of the input projection as red dashed lines and the estimated scaling vector $\hat{t}$ is listed next to the input. It is noteworthy that for regions (a) and (b) only two characteristic facet families can be identified because the third facet family is absent from the crystal morphology (which is correctly identified by the optimization algorithm). For cases where a facet is missing (i.e., regions (a) and (b)) the optimization algorithm returns the value at the boundary of the corresponding region. In the reported exemplary cases, the matching between input and fitted polytope is excellent, and both scale and rotation have been calculated correctly. However, for some rotations of the same polytopes the estimated scaling vector deviates slightly from the input scaling vector. To quantify this effect, we report statistics of the scaling and reconstruction error for all 100 reconstructed particles in the last two columns of this figure. For the scaling statistics, we report the mean of the relative scale $\hat{t}_{i} / t_{i}$ of the $i$ th characteristic facet family as a square. The error bars indicate the standard deviation. The reprojection error in the last column is plotted similarly. Additionally, the morphology distribution is also reported as contour plots in a morphology map, 


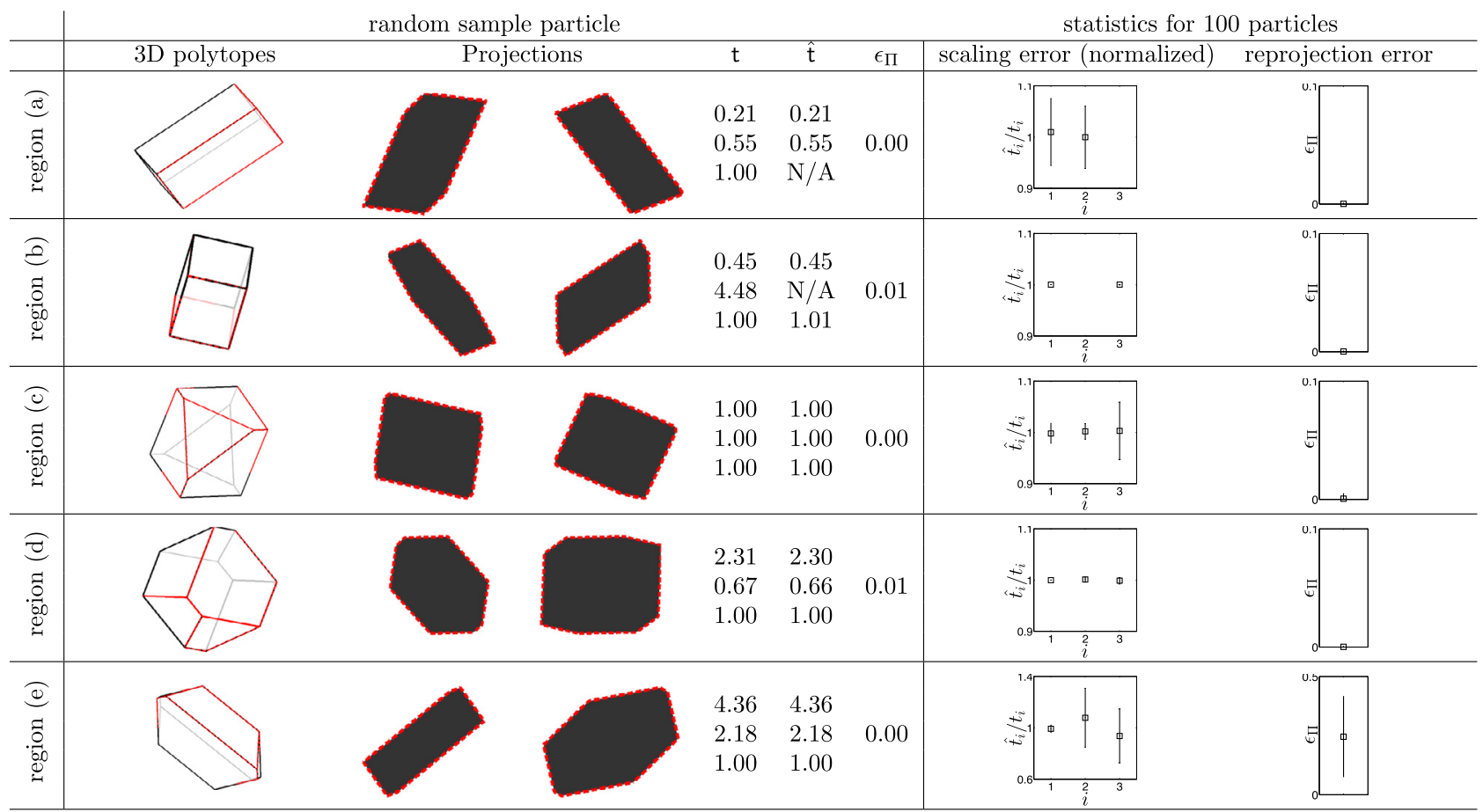

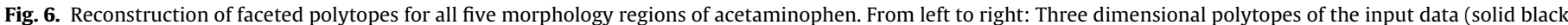

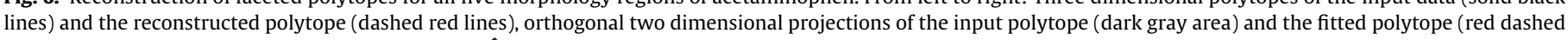

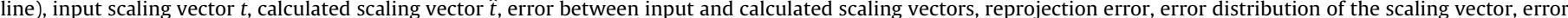

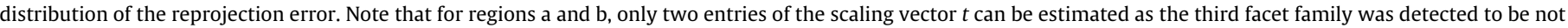
visible. (For interpretation of references to colours in this figure legend, the reader is referred to the web version of this article.)

cf. Fig. 7. The scaling error distribution shows an overall low deviation of the estimated scale as compared to the input. In cases (b) and (d) the optimization result is almost perfect and contour lines mostly overlap with the input. In cases (a) and (c), the deviation does also not exceed $10 \%$ of the input and the mean of the scaling factors is correctly identified. For the platelet-like morphology of region (e) the standard deviation for both the scaling

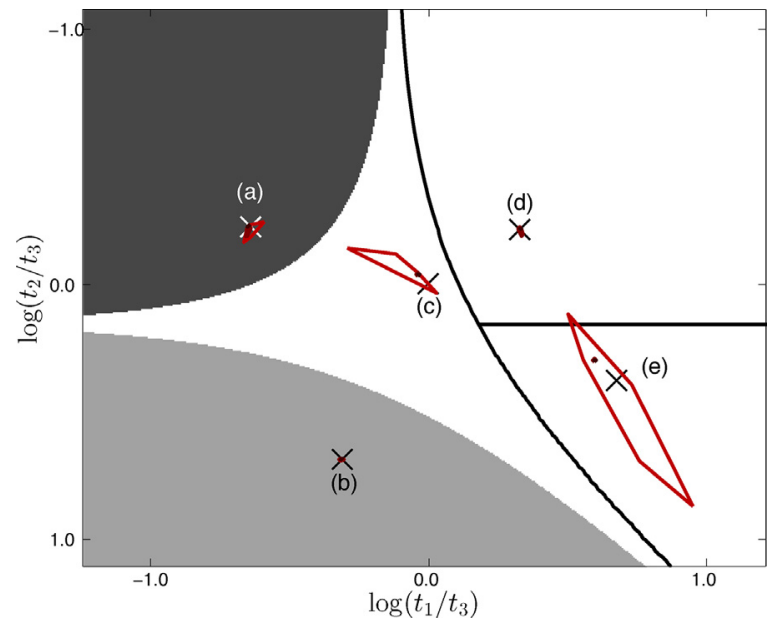

Fig. 7. Zoom in of the morphology map for acetaminophen and the position of the five studied cases of the different morphology regions as black/white crosses. The distributions of the best fitting $\hat{t}$ of the five case studies as indicated in Fig. 6 are plotted as red contour curves into the morphology plane at levels of $1 \%, 2 \%$ and $50 \%$ of the maximum of the distribution. In cases in which only two indices of $\hat{t}$ can be obtained (due to some facets being only virtual), the value of $\hat{t}_{i}$ at the boundary of the morphology cone has been used to calculate the ratio. (For interpretation of references to colours in this figure legend, the reader is referred to the web version of this article.) and the reprojection error is larger, while the mean value is still correctly identified. This result reflects the fact that some morphology regions result in projections that are easy to analyze due to a high number of very characteristic facets, while in other cases the optimization is much more difficult as, depending on the rotation, ambiguous solutions can be found. A clear indication for this behavior is the fact that even for a reprojection error of zero, the scaling error can be considerably larger. However in general, the procedure delivers promising results for the majority of cases studied and even in the worst case the results are still satisfactory, so that we are confident that one may obtain meaningful scaling information.

\subsubsection{Fitting of different crystal systems}

The size calculation procedure can easily be extended to different crystal shapes. Four additional organic crystal systems and a cube are selected as validation cases. The analysis protocol is similar to Section 5.2.1. 100 randomly oriented polytopes of known size have been generated, analyzed, and the results are presented in Fig. 8. In this figure we report the same information as in Fig. 6. Morphology maps, input scaling vectors (as crosses) and distributions of estimated polytopes (as red contour curves) are presented in Figs. 9-11. In general, fitted crystals match the input projections very well, yielding only small reprojection errors. It is worth noting that the error in the scaling vector for all considered facets is less than $3 \%$ in all cases but for $\beta$ L-glutamic acid. However, this might be a result of the very small facets at the tip of the elongated crystal, which are obviously hard to detect in projections, even by the human eye. The reprojection errors are smaller than $3 \%$ in all cases except for the $\alpha$ and $\beta$ polymorph of L-glutamic acid. Even in these worst case scenarios the error is below $8 \%$. Considering the statistics for all 100 projected particles (last two columns), one can see that for all considered particle shapes, with the exception of ascorbic acid crystals, the crystal shape is on average correctly 


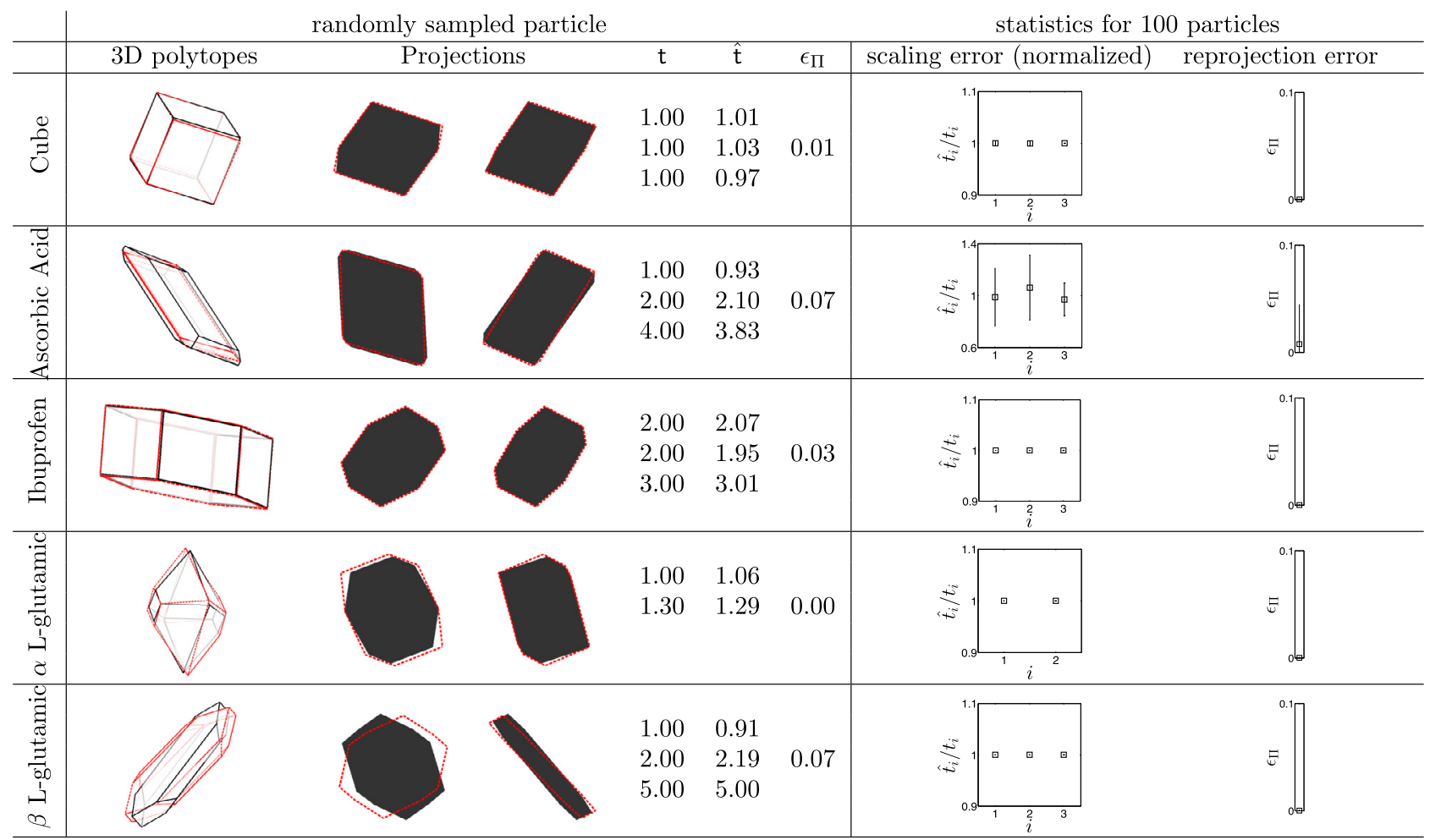

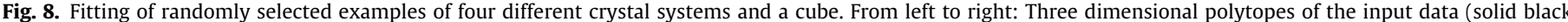

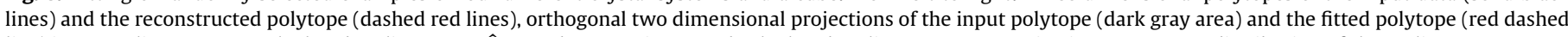

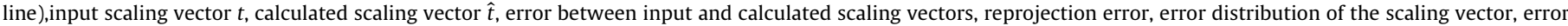
distribution of the reprojection error. (For interpretation of references to colours in this figure legend, the reader is referred to the web version of this article.)

identified and the standard deviation in the identification is negligible, i.e., the results are again almost perfect. For the platelet-shaped ascorbic acid crystal the particle shape is on average also correctly identified, but the standard deviation (just as in the case of the acetaminophen morphology e)) is larger than in the other cases. This is an indication of the fact that the projections of a

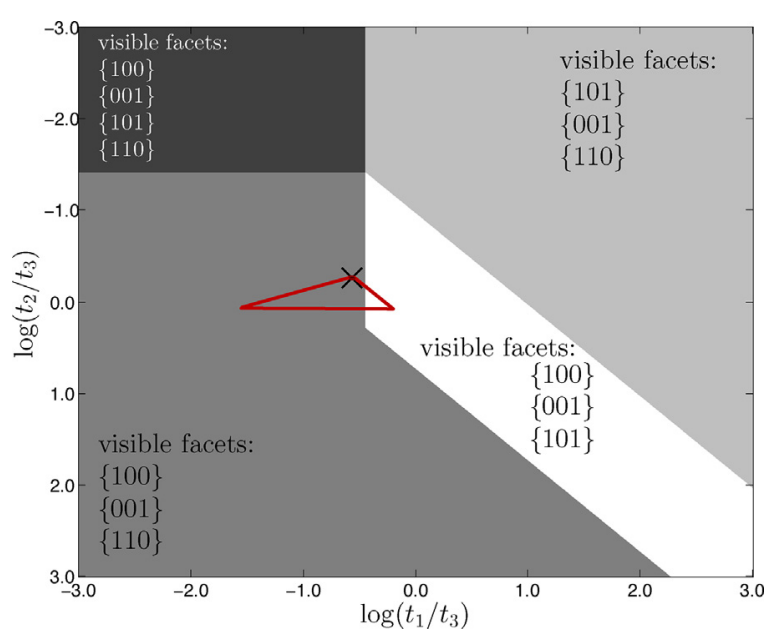

Fig. 9. Morphology map for ascorbic acid where the position of the reconstruction case study is indicated with a black cross. The distribution of the best fitting $\hat{t}$, as calculated and listed in Fig. 8, is plotted as red contour curves into the morphology plane at levels of $1 \%, 2 \%$ and $50 \%$ of the maximum of the distribution. In cases in which only two indices of $\hat{t}$ can be obtained (due to some facets being only virtual), the value of $\hat{t}_{i}$ at the boundary of the morphology cone has been used to calculate the ratio. (For interpretation of references to colours in this figure legend, the reader is referred to the web version of this article.) platelet-shaped object are strongly dependent on its alignment in space. This can result in cases where the vertices that are visible in a projection are not sufficient to uniquely determine the objects rotation, thus yielding non-perfect fits. Overall, these results are encouraging as the algorithm seems to exhibit a good performance for most crystal systems using simulated measurements.

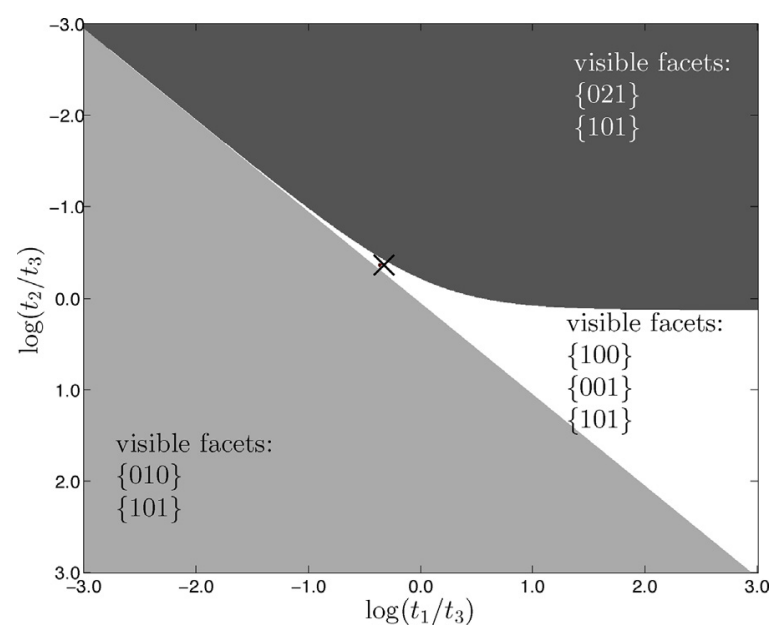

Fig. 10. Morphology map for $\beta$ L-glutamic acid where the position of the reconstruction case study is indicated with a black cross. The distribution of the best fitting $\hat{t}$, as calculated and listed in Fig. 8, is plotted as red contour curves into the morphology plane at levels of $1 \%, 2 \%$ and $50 \%$ of the maximum of the distribution. In cases in which only two indices of $\hat{t}$ can be obtained (due to some facets being only virtual) the value of $\hat{t}_{i}$ at the boundary of the morphology cone has been used to calculate the ratio. Due to the small variance in the reconstruction results in this case, contour lines do mostly overlap. (For interpretation of references to colours in this figure legend, the reader is referred to the web version of this article.) 


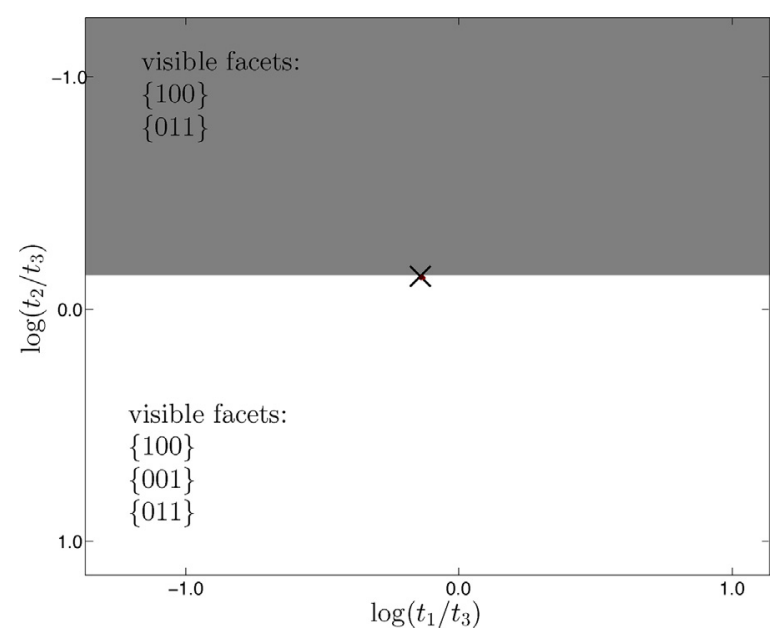

Fig. 11. Morphology map for Ibuprofen where the position of the reconstruction case study is indicated with a black cross. The distribution of the best fitting $\hat{t}$, as calculated and listed in Fig. 8, is plotted as red contour curves into the morphology plane at levels of $1 \%, 2 \%$ and $50 \%$ of the maximum of the distribution. In cases in which only two indices of $\hat{t}$ can be obtained (due to some facets being only virtual), the value of $\hat{t}_{i}$ at the boundary of the morphology cone has been used to calculate the ratio. Due to the small variance in the reconstruction results in this case, contour lines do mostly overlap. (For interpretation of references to colours in this figure legend, the reader is referred to the web version of this article.)

\subsection{Importance of the stereo angle}

In order to assess the potential of the proposed method for observing single crystals in a stereoscopic hot stage setup the importance of the observation angle, i.e., the angle between the two cameras as illustrated in Fig. 12, is investigated. Commercially available stereo microscopes typically have observation angles between $12^{\circ}$ and $14^{\circ}$. However, customized devices could feature stereo angles up to $90^{\circ}$. The change in the reconstruction performance is investigated on the example of an acetaminophen crystal with $t_{i}=1$ for all $i$, i.e., the same case as in Fig. $6 \mathrm{c}$ ). Stereo angles of $12^{\circ}, 14^{\circ}, 30^{\circ}$, $45^{\circ}, 60^{\circ}, 75^{\circ}$ and $90^{\circ}$ have been investigated using 100 randomly orientated particles for each stereo angle. Note that the assumption of random orientation for a compact particle is a valid one, however, one can expect to obtain the same trend (see discussion below) for cases where the particle exhibits a preferential orientation due to its morphology. We are reporting the mean reprojection error and

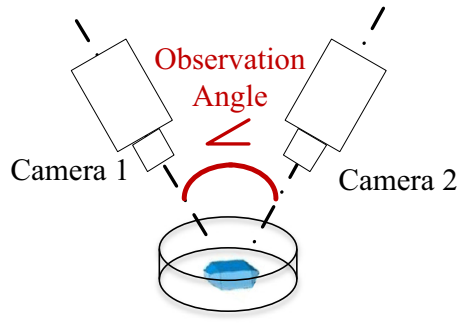

Fig. 12. Explanation of the meaning of the observation angle for stereo microscopic hot stage studies.

its standard deviation in Fig. 13(a). It is apparent from the very low mean reprojection error (square symbols in Fig. 13(a)) that independent of the observation angle, the optimization algorithm is able to find a set of $\hat{R}$ and $\hat{t}$, which is able to match the measured projections. Note that the underlying histograms of reprojection errors for each stereo angle are skewed towards low reprojection errors, so that the lower ends of the error bars (solids lines in Fig. 13(a)) extend below zero. However, when considering the estimated crystal sizes $\hat{t}_{i}$, one can see that neither the accuracy nor the precision of the estimates are satisfactory at low stereo angles, which can be seen in Fig. 13(b) where the exemplary statistics for $\hat{t}_{2}$ are reported. Only for stereo angles greater than $60^{\circ}$ do the precision and the accuracy become satisfactory. This indicates that for small stereo angles, the restoration problem becomes less unique, i.e., the algorithm is likely to find a $\hat{t}$, which is able to match the measured projection data well, but is far away from the actual crystal size $t$. Consequently, the reconstruction of a general faceted crystal from a single projection is a hopeless endeavor. Thus, the take-home message is that a custom made stereoscopic hot stage setup should be built with a stereo angle of at least $60^{\circ}$.

\subsection{Analysis of actual photos of crystals}

The second series of experiments is based on actual photographs of particles taken in the flow through cell, i.e., for orthogonal projections. Crystals of all five previously mentioned organic substances have been prepared according to the following procedure.

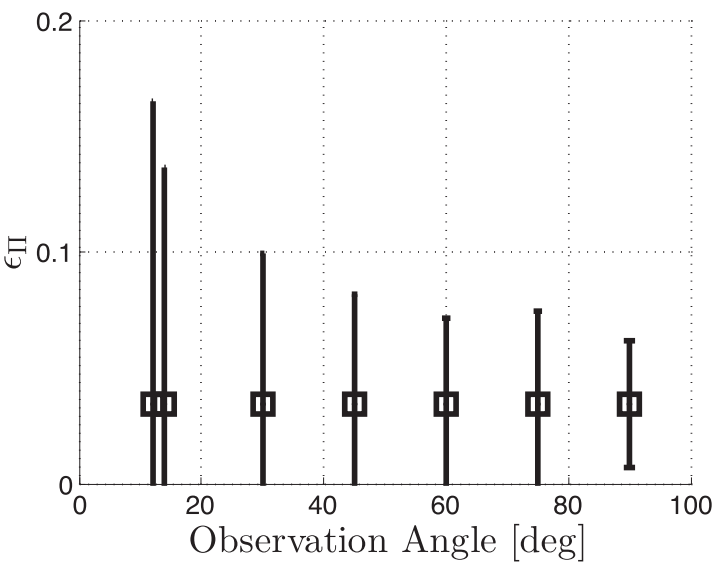

(a)

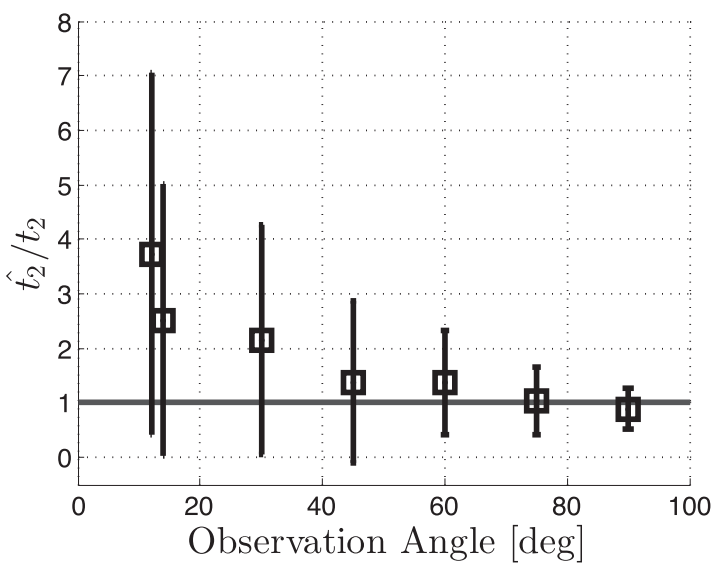

(b)

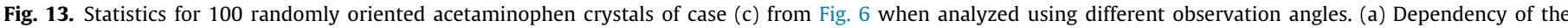

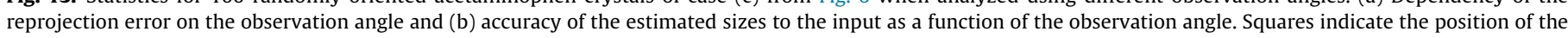
mean value and error bars represent the standard deviation of the distributions. 


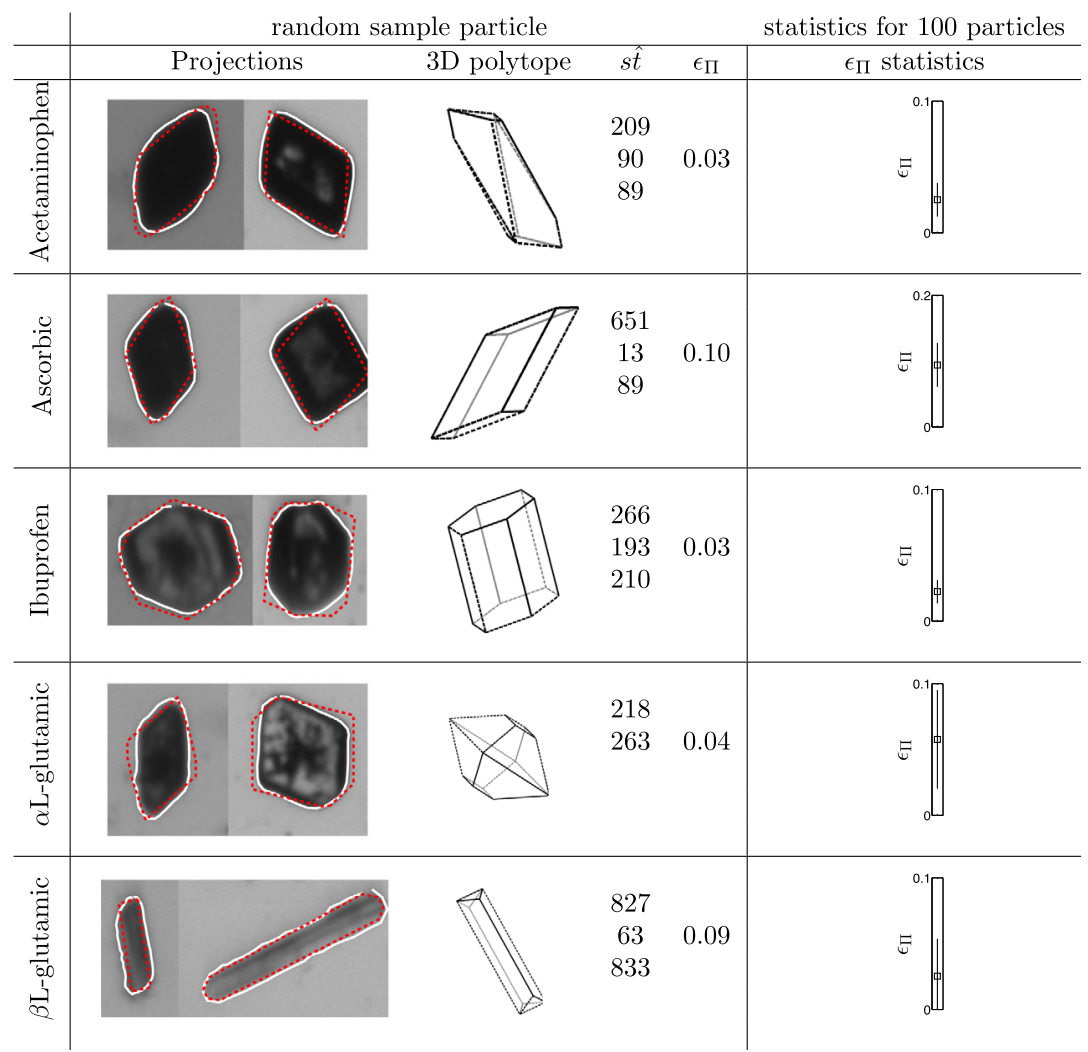

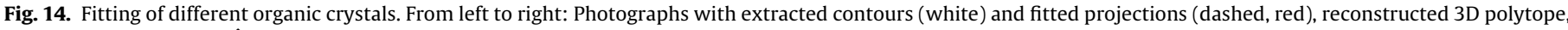
calculated scaling vector $\hat{t}$, reprojection error. (For interpretation of references to colours in this figure legend, the reader is referred to the web version of this article.)

\subsubsection{Preparation of crystals}

acetaminophen (Sigma Aldrich, purity $>98 \%$ ) crystals were obtained by cooling a saturated water solution from $35^{\circ} \mathrm{C}$ to $5^{\circ} \mathrm{C}$ in $6 \mathrm{~h}$, resulting in a suspension that was subsequently wet sieved and dried (Kempkes et al., 2010b). The sieve fraction above $200 \mu \mathrm{m}$ was used for later analysis.

L-Ascorbic acid (Sigma Aldrich, reagent grade) was crystallized from a saturated methanol (Merck Chemicals, purity $>99.9 \%$ ) solution by cooling the mixture and pestled seed from $35^{\circ} \mathrm{C}$ to $5^{\circ}$ in $6 \mathrm{~h}$. The product was wet sieved and analyzed directly afterwards.

Ibuprofen (4-isobutyl- $\alpha$-methylphenylacetic acid, TCI Europe, purity $>98 \%$ ) crystals were prepared by a temperature cycle approach from a saturated mixture of $50 \mathrm{wt} \%$ ethanol (Scharlab, HPLC grade) and $50 \mathrm{wt} \%$ water to which $8 \mathrm{wt} \%$ (solvent basis) Pluronic F127 (Sigma Aldrich, purity $>99 \%$ ) were added. The solution was cooled from $28^{\circ} \mathrm{C}$ to $1{ }^{\circ} \mathrm{C}$ in $3.25 \mathrm{~h}$, heated up to $25^{\circ} \mathrm{C}$ in $2.5 \mathrm{~h}$ and cooled down again to $1^{\circ} \mathrm{C}$ in $4 \mathrm{~h}$. The product suspension was filtered and dried before analyzing.

Crystals of the $\alpha$ polymorph of L-glutamic acid have been precipitated by mixing equimolar amounts of L-glutamic acid monosodium salt hydrate (Sigma Aldrich, purity >99\%) and hydrochloric acid (Fluka, 37\%) in water and continuous stirring for $1 \mathrm{~h}$ at $5{ }^{\circ} \mathrm{C}$ (Cornel et al., 2009). The product suspension was split into two fractions. The first fraction was wet sieved to obtain crystals larger than $125 \mu \mathrm{m}$ which were directly analyzed afterwards. The other half was filtered and dried to be used in a transformation experiment to obtain $\beta$ L-glutamic acid crystals. To this end, a saturated (with respect to the $\alpha$ polymorph) solution of L-glutamic acid monosodium salt hydrate (Sigma Aldrich, purity $>99 \%$ ) and hydrochloric acid (Fluka, $37 \%$ ) in water at $45^{\circ} \mathrm{C}$ was prepared. $\alpha$ crystals were added and the solution was stirred for $48 \mathrm{~h}$ to ensure complete transformation. The product suspension was then directly analyzed.
Water used during experiments was always taken from a MilliQ Advantage A10 system (Merck Chemicals).

\subsubsection{Analysis results for actual photographs}

After particle production was completed, crystals were suspended and photographed in the flow though cell. Images have been processed to extract the sets $D_{1}$ and $D_{2}$ and furthermore $H_{1}$ and $\mathrm{H}_{2}$. Fig. 14 shows exemplary images, fitted contours, reconstructed polytopes, the resulting particle size, the re-projection error for the example case and the reprojection error distribution. As explained before, the calculated scaling vector $\hat{t}$ is multiplied with the scaling constant $s$ thus yielding the size of the reconstructed polytope in $\mu \mathrm{m}$. As the true vectors $t$ are unknown for these crystals, only the reprojection error $\epsilon_{\Pi}$ can be reported. In general, the fitting error is in the same low range as for the simulated images demonstrated in the previous section. The reprojection error is again highest for ascorbic acid, just as in the case of the simulated particles. Additional to the reasons for non-perfect fits given for the simulated particles, one has to realize that real crystals are often not perfectly faceted objects. As such, the particle model may inherently not be able to represent crystals that have been photographed in the flow through cell. Additional noise is introduced due to dust on the images, errors in the contour extraction, a limited resolution, and a limited depth of field. Despite these limitations, the fitting results appear to be generally satisfying enough to extract useful facet information form the reported images.

\subsubsection{Crystal growth}

Finally, we want to investigate the possibility of applying the image analysis and polytope recognition procedure to a dynamic experiment in which crystals grow. A population of acetaminophen crystals is exposed to supersaturation during cooling crystallization 

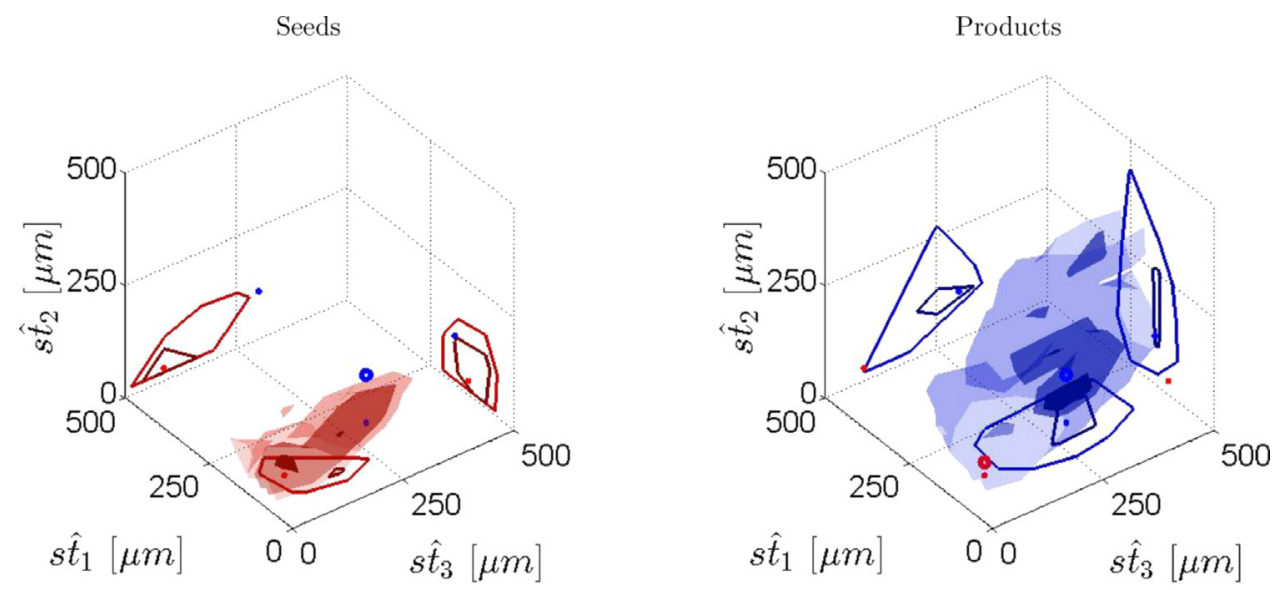

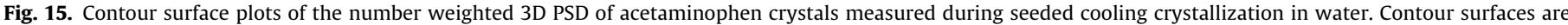

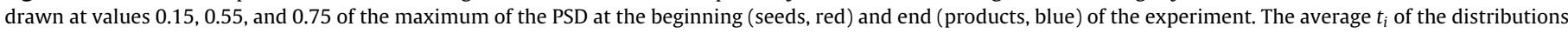

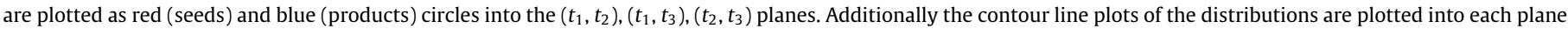
at values of 0.05 and 0.085 . (For interpretation of references to colours in this figure legend, the reader is referred to the web version of this article.)

in water, and images of particles in suspension are continuously recorded for the entire duration of the experiment.

Acetaminophen seeds were produced by rapidly cooling a saturated solution of acetaminophen and water (Sigma Aldrich, purity 98.0\%) from $40^{\circ} \mathrm{C}$ to $10^{\circ} \mathrm{C}$. The product was filtered, dried, and sieved. The sieve fraction between 125 and $250 \mu \mathrm{m}$ was used. Crystals have been suspended in a saturated solution of acetaminophen and water at $30^{\circ} \mathrm{C}$ at a suspension density of $1 \mathrm{~g} / \mathrm{kg}$. The resulting suspension was then cooled to $25^{\circ} \mathrm{C}$ with a cooling rate of $0.5^{\circ} \mathrm{C} / \mathrm{h}$. The temperature was subsequently kept constant for an additional $200 \mathrm{~min}$.

The low cooling rate (and therefore low supersaturation level) causes the aspect ratio of the acetaminophen crystal population to increase, which is in agreement with previous observations (Kempkes et al., 2010a). With reference to Fig. 2 the vast majority of particles was measured in region (c). The change of the distribution from the seed crystals (red) to the product crystals (blue) is shown as isosurface plots and isocontour plots in Fig. 15. Note that information during the cooling crystallization experiment was recorded but not analyzed due to a lack of computational power, because the reconstruction of one crystal of typical complexity (i.e., obtaining a solution of the nonlinear problem Eq. (6) from all warm start points) takes roughly $1 \mathrm{~min}$. However, in order to obtain a smooth PSSD the analysis of several thousand particles is necessary, thus resulting in several days of computational time. The identical experiment has been presented before in (Schorsch et al., 2014) using a different type of particle model. In contrast to the generic particle analysis the polytope based approach delivers very scattered distributions. It is noticeable that the movement of the center of the PSSD is only due to an increase in the scaling factors $s$ and cannot be attributed to a dominant change in $t$, i.e., the before-mentioned increase in aspect ratio could not be clearly identified. Since the computational cost of the polytope reconstruction in comparison to the generic particle approach is high, a thorough comparison between the two methods based on idealized pictures seems to be warranted and is presented in the following section.

\subsection{Comparison to generic particles}

In previous works (Schorsch et al., 2012, 2014) we have reported a set of generic particle models to describe crystals. These models are spheres, cylinders and cuboids; as such these generic particles are able to cover a wide range of prototypical shapes. The size information in this case is computed from geometric information that is available in a stereoscopic setup, but without directly minimizing the reprojection error. Clearly, the additional computational effort of the polytope reconstruction procedure needs to be justified. A direct comparison between the two techniques is difficult because the output of the two models is quite different (generic particle vs. faceted polytope). Hence, we compare the two most important macroscopic properties of the crystals, i.e., their aspect ratio (the ratio of maximum to minimum size) and the particle volume. The analysis is performed using an exemplary particle of each of the previously mentioned organic substances and a cube-shaped particle, which were randomly oriented 100 times (each). The input size vectors, estimated volumes and aspect ratios, as well as the true aspect ratio are reported in Table 8 for each of these cases. The outcome of this comparison depends on the chosen inputs, i.e., one may find conditions for which either approach is better. Therefore, we have chosen cases which cover a reasonable range of different geometries.

For the needle-like $\beta$ glutamic acid and the cubes, there is no advantage in using the polytope model because the shape gets almost perfectly matched in all cases independent of the

Table 8

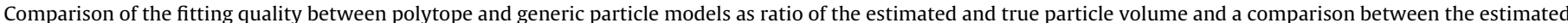
and true aspect ratio (AR) as mean and standard deviation of 100 repetitions of fitting the respective crystal to the polytope particle and generic particle model each.

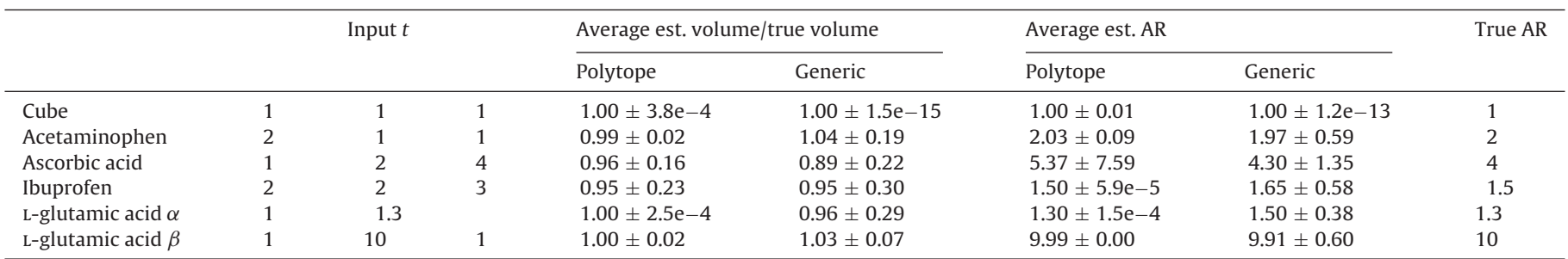


particle model. For the other cases in average the generic case delivers a reasonable estimation. However the standard deviation of the underlying recovered distribution is wider than for the polytope reconstruction, which means that generic models sometimes over- or underestimate the particle size due to an inherent mismatch between generated particle and fitted shape. The platelet-like ascorbic acid crystals are not captured well by either particle model, which is not surprising for the reasons mentioned already above. It is worth noting that the set of generic particle shapes can also be easily extended to get a better model representation for a specific crystal shape if required, so as to improve the reconstruction accuracy, while preventing an increased computational effort.

Calculating the faceted shape of a crystal requires particles of a certain size or an imaging system with an excellent resolution that equals the quality of a microscope in order to have sufficient contour data to solve the polytope reconstruction problem. It also requires crystals of a very high quality, i.e., crystals without damaged vertices, etc., to obtain meaningful results. Given that many crystals obtained from a real crystallizer do exhibit some kind of imperfection, the advantage that a polytopic model describes the crystal morphology of primary particles on a first principles level vanishes when moving from idealized generated images to crystals produced in real crystallization experiments. The generic particle classes on the other hand are more robust and deliver good estimates of the most important macroscopic properties (aspect ratio and volume of the crystal) at a much lower computational effort.

\section{Concluding remarks}

An optimization-based approach is applied to reconstruct the faceted morphology of crystals from its two stereoscopic projections. These projections are obtained from two different but coplanar observation directions. To investigate the influence of the geometry of the measurement device and the crystal's shape, we have varied the angle between the two coplanar observation directions (the so-called stereo angle) and the orientation and morphology of the analyzed particles. Using simulated images, we have demonstrated that the approach works satisfactorily with a stereo angle of $90^{\circ}$ by using simulated images of crystals of five organic substances. In these cases, the procedure delivers nearly perfect reconstructions of the input projections, except for rare cases where particles are seen from unfavorable angles where only an insufficient number of vertices is visible or where the facets themselves were rather small. A rotational dependence of the reconstruction quality is especially noticeable for platelet shaped particles. However, even in these worst cases the error in the estimated sizes never exceeded $10 \%$.

In our investigation of different stereo angles, we have found that a minimum of $60^{\circ}$ is required in order to obtain trustworthy facet size information using our optimization based approach. It can be argued that the information content of the two projections decreases as the stereo angle becomes smaller, so that a number of different orientations and size vectors yield indistinguishably similar projections when they are compared to the measured projections. Consequently, a stereoscopic hot-stage apparatus that can be used to obtain high quality information about the faceted morphology of a crystal should be constructed with a stereo angle of at least $60^{\circ}$. Comparing calculated reprojections with photographs of crystals from a real crystallization process (obtained using the setup presented in Schorsch et al. (2014)) yields a satisfactory agreement with respect to the reprojection error as well. However the correctness of the estimated facet sizes appears questionable, since a shape change of the crystals that occurred in a cooling crystallization experiment of Acetaminophen crystals, that was clearly identifiable using generic model particles and by a visual comparison of initial and final particles, could not be identified anymore. We believe that the growing crystals from a real crystallization experiment carried out in a crystallizer are often of too low quality to accurately and convincingly reconstruct a faceted morphology from two projections obtained of such particles. Therefore, we find that, at least for an on-line measurement technique such as the flow through cell, generic particle models yield a reasonable estimate of shape and size distributions for many applications at a much lower computational cost that enables the use of the measurement device for control purposes. Nevertheless, we think that a possible area of application for the proposed polytopic reconstruction method could be in hot stage microscopy where the crystals grown are typically of higher quality with fewer imperfections.

\section{Acknowledgement}

The authors are thankful to the Swiss National Science Foundation for their support (project number 200021-135218).

\section{References}

Ahmad OS, Debayle J, Gherras N, Presles B, Févotte G, Pinoli J-C. Quantification of overlapping polygonal-shaped particles based on a new segmentation method of in situ images during crystallization. J Electron Imaging 2012;21:021115.

Borchert C, Sundmacher K. Morphology evolution of crystal populations: modeling and observation analysis. Chem Eng Sci 2012:70:87-98.

Borchert C, Sundmacher K. Efficient formulation of crystal shape evolution equations. Chem Eng Sci 2013;84:85-99.

Bradski G. The OpenCV Library. Dr. Dobb's journal of software tools; 2000.

Calderon De Anda J, Wang X, Lai X, Roberts K. Classifying organic crystals via inprocess image analysis and the use of monitoring charts to follow polymorphic and morphological changes. J Process Control 2005;15:785-97.

Cano H, Gabas N, Canselier JP. Experimental study on the ibuprofen crystal growth morphology in solution. J Cryst Growth 2001;224:335-41.

Cornel J, Lindenberg C, Mazzotti M. Experimental characterization and population balance modeling of the polymorph transformation of L-glutamic acid. Cryst Growth Des 2009;9:243-52.

Davey R, Fila W, Garside J. The influence of biuret on the growth kinetics of urea crystals from aqueous solutions. J Cryst Growth 1986;79:607-13.

Douglas DH, Peucker TK. Algorithms for the reduction of the number of points required to represent a digitized line or its caricature. Int J Geograph Inf Geovis 1973;10:112-22.

Eggers J, Kempkes M, Mazzotti M. Measurement of size and shape distributions of particles through image analysis. Chem Eng Sci 2008a;63:5513-21.

Eggers J, Kempkes M, Mazzotti M. Monitoring size and shape during cooling crystallization of ascorbic acid. Chem Eng Sci 2008b;64:163-71.

Graham RL. An efficient algorithm for determining the convex Hull of a finite planar set. Inform Process Lett 1972;1:132-3.

Hirokawa S. The monoclinic form of $p$-hydroxyacetanilide. Acta Crystallogr Sect B 1976;32:1283-5.

Hirokawa S. A new modification of L-glutamic acid and its crystal structure. Acta Crystallogr 1955;8:637-41.

Hours JH, Schorsch S, Jones CN. Parameteric polytope reconstruction, an application to crystal shape estimation. IEEE Trans Image Process 2014:23:4474-85.

Huttenlocher DP, Klanderman GA, Rucklidge WJ. Comparing images using the hausdorff distance. IEEE T Pattern Anal 1993;15:850-63.

Hornegger J, Tomasi C. Representation issues in the ML estimation of camear motion. In Proc 7th IEEE Int Conf Comput Vis 1999;1:640-7.

Hvoslef J. The crystal structure of L-ascorbic acid, 'vitamin C'. I. The X-ray analysis. Acta Crystallogr Sect B 1968;24:23-35.

Kempkes M, Eggers J, Mazzotti M. Measurement of particle size and shape by FBRM and in situ microscopy. Chem Eng Sci 2008;63:4656-75.

Kempkes M, Vetter T, Mazzotti M. Measurement of 3D particle size distributions by stereoscopic imaging. Chem Eng Sci 2010a;65:1362-73.

Kempkes M, Vetter T, Mazzotti M. Monitoring the particle size and shape in the crystallization of paracetamol from water. Chem Eng Res Des 2010b;88:447-54.

Kitamura M, Ishizu T. Growth kinetics and morphological change of polymorphs of L-glutamic acid. J Cryst Growth 2000;209:138-45.

Kuvadia ZB, Doherty MF. Spiral growth model for faceted crystals of noncentrosymmetric organic molecules grown from solution. Cryst Growth Des 2011;11:2780-802

Kvasnica M, Grieder P, Baotic M. Multi-parametric toolbox (MPT); 2012 http://control.ee.ethz.ch/ mpt/

Lakhtin A, Ushakov V. Minimization of the hausdorff distance between convex poly hedrons. J Math Sci 2005:126:1553-60.

Larsen PA, Rawlings JB, Ferrier N. Model-based object recognition to measure crystal size and shape distributions from in situ video images. Chem Eng Sci 2007;62:1430-41. 
Larsen PA, Rawlings JB. The potential of current high-resolution imaging-based particle size distribution measurements for crystallization monitoring. AIChE J 2009;55:896-905.

Larsen PA, Rawlings JB, Ferrier N. An algorithm for analyzing noisy, in situ images of high-aspect-ratio crystals to monitor particle size distribution. Chem Eng Sci 2006;61:5236-48.

Lovette MA, Browning Robben A, Griffin DW, Sizemore JP, Snyder RC, Doherty MF. Crystal shape engineering. Ind Eng Chem Res 2008;47:9812-33.

MATLAB. Version 7.13 (R2011b). Natick, Massachusetts: The MathWorks Inc; 2010

Patience DB, Rawlings JB. Particle-shape monitoring and control in crystallization processes. AIChE J 2001;47:2125-30.

Schmidt J, Niemann H. Using quaternions for parametrizing 3D rotations in unconstrained nonlinear optimization. In: Proc vis modeling vis conf (VMF); 2001. p. 399-406, Available online: http://dl.acm.org/citation.cfm?id=647260.718651

Schorsch S, Ochsenbein DR, Vetter T, Morari M, Mazzotti M. High accuracy online measurement of multidimensional particle size distributions during crystallization. Chem Eng Sci 2014;105:155-68.

Schorsch S, Vetter T, Mazzotti M. Measuring multidimensional particle size distributions during crystallization. Chem Eng Sci 2012;77:130-42.

Shankland N, Wilson C, Florence AJ, Cox P. Refinement of Ibuprofen at $100 \mathrm{~K}$ by single-crystal pulsed neutron diffraction. Acta Crystallogr Sect C 1997;53:951-4.
Singh MR, Ramkrishna D. A comprehensive approach to predicting crystal morphology distributions with population balances. Cryst Growth Des 2013;13:1397-411.

Srinivasan K, Dhanasekaran P. Separation and nucleation control of $\alpha$ and $\beta$ polymorphs of L-glutamic acid by swift cooling crystallization process. Amino Acids 2011;40:1257-60.

Srinivasan K, Vanitha Devi K. Characterization of L-ascorbic acid single crystals grown from solution with different solvents. Cryst Res Technol 2010;45:946-52.

Variankaval N, Cote AS, Doherty MF. From form to function: crystallization of active pharmaceutical ingredients. AIChE J 2008;54:1682-8.

Wächter A, Biegler LT. On the implementation of a primal-dual interior point filter line search algorithm for large-scale nonlinear programming. Math Program 2006; 106:25-57.

Wang XZ, Calderon De Anda J, Roberts K. Real-time measurement of the growth rates of individual crystal facets using imaging and image analysis: a feasibility study on needle-shaped crystals of L-glutamic acid. Chem Eng Res Des 2007; 85: $921-7$.

Wang X, Roberts K, Ma C. Crystal growth measurement using 2D and 3D imaging and the perspectives for shape control. Chem Eng Sci 2008;63:1173-84.

Zhang Y, Sizemore JP, Doherty MF. Shape evolution of 3-dimensional faceted crystals. AIChE J 2006;52:1906-15. 